\title{
The Application of Computers to the Classification of Streptococci
}

\author{
By G. COLMAN \\ Wright-Fleming Institute, St Mary's Hospital \\ Medical School, London, W. 2
}

(Accepted for publication 8 July 1967)

\begin{abstract}
SUMMARY
Computers were used for numerical studies as an aid to the classification of streptococci. The results obtained with 75 biochemical and physiological tests of 2 I 6 strains were examined by three different computer programmes. Two of these programmes formed clusters in general agreement with the wellestablished species and also formed three clusters among the viridans-like streptococci. These 3 clusters seem to provide a basis for the recognition of 3 divisions among the streptococci which are at present usually described as lacking adequate distinguishing characters.
\end{abstract}

\section{INTRODUCTION}

The streptococci form a group of bacteria of considerable medical and veterinary importance yet, with the exception of one or two species, their classification is very unsatisfactory. There has been a tendency to attribute over-riding importance to a single character, namely the so-called group antigen, but various workers have shown that this may lead to the allocation to one taxon of quite disparate organisms as, for example, among strains of serological group $\mathrm{K}$ (Williams, I956), group $\mathrm{M}$ (Skadhauge \& Perch, 1959), and group D (Deibel, I964). It seems that a satisfactory classification will be achieved only by taking into account several different characters, and attempting to define taxa on the basis of overall similarity of the constituent strains. In an attempt to unravel some of the taxonomic problems of this genus, a collection of some 350 strains of streptococci was assembled and subjected to a large number of tests.

Numerical taxonomy appeared to offer a good method for examining the collection of strains, even if, with Mayr (1965), one does not necessarily accept the thesis that one is bound to analyse the occurrences of large numbers of attributes chosen at random, or to accept machine-formed clusters as the final outlines of taxa. The first analysis of 216 strains, by using a programme devised by Rogers \& Tanimoto (I960), yielded clusters which often conflicted seriously with those recognized by tradition or intuition. The same material was subsequently examined with two other programmes, which yielded clusters that are reasonably conformable one with the other, and are intuitively more 'satisfactory'. This paper reports an outline of the classification of streptococci as derived from this numerical analysis and a comparison of the classifications indicated by the use of the three different computer programmes. 


\section{METHODS}

Strains examined. The strains in the collection do not represent any natural population, but were selected to give as wide a representation as possible of strains which have in the past been given more or less specific names, and to include a large number of strains that cannot at present be adequately classified. Some of the strains were from culture collections but many were freshly isolated; most of the strains were of human origin.

A total of 216 strains is included in this study: 203 streptococci, Io aerococci, 2 leuconostocs, and I pediococcus. The attributes of the aerococci included have been given in detail elsewhere (Colman, 1967).

Reference numbers for each strain are given in Table 2. The numbers on the extreme left of the table are the catalogue numbers of the strains. The letters NCTC show the strains supplied by the National Collection of Type Cultures, Colindale, the letters FW indicate that the strains were, with the exception of those numbered $1,5,6,7,9$, I3, 15, 16, 32, 34, 35, 36, 37, 49, and 250, isolated at the Wright Fleming Institute. The prefix RB is given to strains isolated by Dr K. B. Rogers, Birmingham. s, with the exception of S I230 and S I545, indicates strains from the collection of Professor M. Seelemann formerly of Kiel, and BU indicates strains from the collection of the late Dr Coralie Baldovin-Agapi of Bucharest. Numerous other workers generously sent strains for examination and as far as possible their numbers are used to denote the strains they sent; these strains make up the rest of the table.

Bacteriological tests. This study is based on the results from a total of 75 tests on each strain as listed in Table $\mathbf{I}$.

One of the computer programmes, the single-linkage method, allows tests to be considered as 'qualitatives' or 'quantitatives' as well as 'dichotomies'. With this programme the tests scored as qualitatives are: arrangement in glucose broth; changes in horse blood agar; colonial form on sucrose agar. Tests scored as quantitatives are: ability to grow on bile agar; growth in $\mathrm{NaCl}$ broth; growth in tellurite broth; presence of trace or readily detectable amounts of rhamnose in cell-wall hydrolysates. All these tests are scored as dichotomies in the other programmes.

\section{The programmes used}

Single-linkage method. The programme for this method was prepared by G. J. S. Ross of The Rothamsted Experimental Station and is a version in I.C.T. Orion machine language of J. C. Gower's programme originally prepared for the Elliott $40 \mathrm{I}$ digital computer. All procedures with this programme were carried out by $\mathrm{Mr}$ Gower and other members of the staff of the Rothamsted Experimental Station. The tests with this programme are of three kinds and are called dichotomies, qualitatives and quantitatives. Dichotomies are tests in which the response is binary, for example, the response of the indicator in the test for the production of acid from lactose is recorded as either + or - . Qualitatives are tests with mutually exclusive attributes such as the changes produced in the blood agar beneath the colonies. These are recorded as one of the following: haemolytic, greening, indifferent. Quantitative tests are those which form a series such as the ability to grow in $4 \%$ and $6.5 \% \mathrm{NaCl}$ broth.

These different sorts of tests affect the coefficient of similarity in different ways. 
Dichotomies are scored as follows. When lactose is fermented by two strains, $i$ and $j$, in calculating the similarity coefficient of these two strains there is a count of $\mathrm{I}$ in both numerator and denominator. When lactose is fermented by only one strain there is a count of $I$ only in the denominator. When lactose is not fermented by either strain there is no score in either numerator or denominator. This method of scoring does not take a negative match as a similarity; the arguments for and against this procedure were discussed by Sneath (1962). In the scoring of qualitative tests, when

\section{Table I. The bacteriological tests used in this work}

Arrangement in glucose broth: pairs; chains

Changes in horse blood agar: haemolysis; greening; indifference ( $=$ no change)

Serological grouping procedures (23 sera): Lancefield groups A, B, C, D, E, F, G, H, $\mathrm{K}, \mathrm{L}, \mathrm{M}, \mathrm{N}, \mathrm{O}, \mathrm{P}, \mathrm{Q}, \mathrm{R}, \mathrm{S}$, and T; Ottens and Winkler types I, II, III, IV, and V (Williams, 1958)

Bile solubility (Anderson \& Hart, 1934)

Optochin sensitivity ( $5 \mu \mathrm{g}$. disc)

Bacitracin sensitivity $(0 \cdot 1 \mathrm{U}$. disc)

Nitrofurazone sensitivity $(10 \mu \mathrm{g}$. disc)

Growth on bile agar: $10 \%$ bile; $40 \%$ bile (Williams, 1958)

Growth on MacConkey agar

Growth in salt broth: $4 \% \mathrm{NaCl} ; 6.5 \% \mathrm{NaCl}$

Survival after heating at $60^{\circ}$ for $30 \mathrm{~min}$.

Growth at $45^{\circ}$

Ammonia from arginine

Colonial forms on $5 \%$ sucrose agar: colonies without special features; fleshy colonies; adherent colonies; watery colonies

Hydrolysis of hippurate (Ayers \& Rupp, 1922; sulphuric acid reagent)

CAMP test (Munch-Petersen \& Christie, 1947)

Production of hyaluronidase (Henriksen \& Joner, 1962)

Production of $\beta$-glucuronidase (Williams, 1954)

Acetoin produced from glucose ( $\alpha$-naphthol reagent)

Hydrolysis of starch (Andrewes, 1930)

Splitting of aesculin (ferric citrate as indicator)

Production of acid from fermentable substances (phenol red as indicator): glucose; maltose, glycerol (aerobically); glycerol (anaerobically); mannitol; sorbitol; arabinose; lactose; sucrose; trehalose; raffinose; salicin; inulin

Detection of cell-wall residues: glycerol; anhydroribitol; rhamnose readily detectable; rhamnose present in trace amounts; glucose; galactose; galactosamine; fucosamine (Colman \& Williams, 1965)

Growth in tellurite broth: $0.1 \%$ potassium tellurite; $0.025 \%$ potassium tellurite; $0.01 \%$ potassium tellurite

the strains are both haemolytic there is a score of $\mathrm{I}$ in both numerator and denominator, but there is no score in either numerator or denominator when one strain is greening and the other haemolytic. In the scoring of quantitative tests there is a range of 2 when measuring the ability to grow in $\mathrm{NaCl}$ broth, and there is a count of $\mathrm{I}$ in both numerator and denominator when both strains grow in $6.5 \% \mathrm{NaCl}$ broth, and a count of $\frac{1}{2}$ in the numerator and $\mathrm{I}$ in the denominator when one strain grows only in $4 \%$ and the other in both 4 and $6.5 \% \mathrm{NaCl}$ broth.

The 'similarity coefficient' of two strains is obtained by dividing the sum of the scores of similarity by the number of relevant tests. The similarity coefficients are sorted by the machine to produce the clusters. The strains are first examined for any that are identical; there were no completely identical pairs of strains in our collection. The collection of similarity coefficients is then examined for strains similar 
at the $97.5 \%$ or a higher level. In our collection 3 pairs of strains were linked at this level of similarity: the strains in the array in Table 2 numbered 9 and 10,95 and 96, and 159 and 160 . Restricting our attention to the first pair, no further strains were added at the $95 \%$ level, but at the $92.5 \%$ level a third strain, numbered 8 , was added. At the $90 \%$ level of similarity, 3 more strains were added to this cluster, making at this level of similarity a cluster of 6 strains. By this method each strain will eventually join a cluster and the clusters will eventually join together until all the strains in the collection form one cluster.

The Harrison method. This programme was prepared by P. J. Harrison of Imperial Chemical Industries, Wilmslow, originally for the classification of chemicals. (Harrison 1968). Our data were given to Mr Harrison, and he and other members of the staff of I.C.I. did all the computer procedures.

In this programme all tests are treated as dichotomies and the probabilities of the attributes not being randomly distributed are calculated. In the calculation of the similarity of a pair of strains, a weighting of 2 was given to positive matches and a weighting of $\mathrm{I}$ to the negative matches and mismatches. The 'expected' distribution of attributes is calculated from the matrix of similarities and this serves as a reference for calculating the odds that a particular combination of attributes could arise by chance. For each probability level in turn the largest cluster is found, and then the next largest cluster with the following conditions: that a strain previously clustered is not to be the centre of a new cluster, at least one-half of the strains in the new cluster are to be strains not previously clustered, and all clusters must contain at least five strains. The programme is repeated while clusters can be formed that fulfil these conditions.

Table 2 contains the clusters formed at the 99 and $80 \%$ significance levels. As would be expected, not all strains in the collection were clustered, and some strains appeared in more than one cluster.

The Rogers \& Tanimoto method. Rogers \& Tanimoto (I960) described a method for the classification of plants and International Business Machines, London, made available to us a version of this programme (I.B.M. 7090/94 Taxonomy Application) which was run on the I.B.M. 7090 digital computer in the Centre for Computing and Automation at Imperial College. All tests were examined as dichotomies.

The computer first determines the similarity coefficients of all pairs of strains by dividing the number of attributes possessed in common by a pair of strains by the number of attributes possessed by either of them. The computer then makes a count for each strain of the number of strains with at least one attribute in common. The results of this count are used to provide the primary ranking of the strains. A strain with something in common with 100 other strains ranks above a strain with something in common with 99 other strains. As more than one strain may have something in common with 100 other strains the computer then performs a secondary ranking of the strains. This is done by multiplying together all the non-zero values of the similarity coefficients, using the negative of the binary logarithm. The logarithms of numbers less than I but greater than o are negative in sign, and the logarithm of the number increases in magnitude as the number becomes smaller. The strain that is ranked first among those having something in common with 100 other strains is the strain with the lowest sum. A geometrical analogy was used by Rogers \& Tanimoto (I960) and they likened strains to points in space and similarity to distance, like strains 
being separated by short distances. Multiplying together similarities and choosing the strain with the lowest sum should lead to the selection of a strain in the centre of a cluster as the strain at the head of the list, or the prime strain.

The cluster is formed from the results of the primary and secondary ranking of the strains. The strain at the head of the list is the centre or prime strain of the cluster and the second strain in the list provides the boundary. The cluster is formed from those strains which have a similarity coefficient, relative to those ranked first, greater than or equal to those ranked second. The strains forming the cluster are not further considered and the process of clustering is repeated while there are two or more strains remaining unclustered.

The size of the cluster is thus determined by the similarity of the second strain in the ranking to the prime strain. When the strain ranked second is very similar to the strain ranked first the cluster will be of two specimens only, or the second strain may provide the boundary for a homogeneous cluster. When the second strain is dissimilar the cluster formed will contain many specimens with a wide range of similarity to the prime strain. All of these possibilities occurred.

The whole process was repeated three times with deletions of the most common attributes and the large clusters were also separately studied as discrete populations. The results of these additional runs did not appear to add anything to the results of the first examination of our strains, using all of the attributes. It is the result of the latter study that is given in Table 2.

\section{RESULTS}

The programmes

The table. The results of the computer studies are summarized in Table 2. Reading from the left the first column gives the catalogue number of the strain. The second column lists the serological group, if any, and also the specific name when this is well established; otherwise the appearance of the colonies on blood agar plates is listed. Changes in blood agar do not form an adequate basis for classification but they are useful descriptive characters frequently used in medical microbiological laboratories. The diagram is a summary of the results with the single linkage method and it shows the similarity level (at $75 \%$ or more) at which the strains are clustered. The first two strains in Table 2 are linked at the $90 \%$ level, which means that the coefficient of similarity of these two strains is equal to or greater than $90 \%$ but less than $92.5 \%$. A third strain is added at $77.5 \%$ and this similarity could be with one or both of the first two strains; this strain appears third on the table because it was by chance further down the list of strains fed into the computer.

The series of numbers, I to 216 , to the right of the diagram is the order in which the strains were printed out from the computer in the single-linkage method. It is these numbers which are referred to throughout this paper. Further to the right of Table 2 are the results obtained with the two other programmes. The strains in the clusters formed by these programmes have been re-arranged, within the clusters, to agree as closely as possible with the order of the strains in the single linkage method. An asterisk is used when a strain can be placed in the same relative position as it has with the single-linkage method. When strains cannot be aligned in this way the numbers of the strains in the single-linkage method are used to indicate the composition of the clusters. 
Single-linkage method. In this method all strains were brought into a single cluster at $45 \%$ overall similarity, and 208 of the 216 strains were formed into one cluster at the $65 \%$ level. The limits of the smaller clusters formed at higher similarities and discussed below were not solely machine-determined but were also influenced by subsequent inspection. For instance, one could choose any one of the four strains, I05, I08, I I2, or I16, as the lower limit of the cluster that began with strain 88 .

The admission of a new strain to a cluster, at perhaps the $90 \%$ level will occur when its similarity to any strain in the cluster is $90 \%$ or higher, as is indicated by the namesingle-linkage method. Sokal \& Sneath (I963) suggested that this method could lead to the linkage of two clusters through the similarity of two strains, one in each cluster, the other members of the cluster being more dissimilar. Something of this sort appears to have occurred with our material. Strains 4 I, 42, and 43 (indifferent streptococci) are seen in Table 2 to come between strains 37 to 40 and 44 to 48 , all of these nine being salivarius strains. The two separate clusters of salivarius are joined at the $80 \%$ level by the linkage of strains 39 and 45 , and they are joined to the cluster bounded by strains 4 and 28 by the similarity at the $80 \%$ level of strains $I 7$ and 46 . The strains $4 \mathrm{I}, 42$, and 43 separate the salivarius strains because at the $80 \%$ level these too are linked to the large cluster and these three strains were listed in the data after the salivarius strains 37 to 40 and before the salivarius strains 44 to 48 .

The Harrison method. At the $99 \%$ probability level, 87 strains were formed into 7 clusters, and at the $80 \%$ level I 30 strains were formed into 9 clusters.

The Rogers \& Tanimoto method. When all attributes were used in the analysis 17 clusters were formed, but as can be seen from Table 2 the composition of the clusters appears to be unrelated to the conventional classification of the streptococci. Therefore, the presentation of the results with this programme is restricted to a listing, in Table 2, of the composition of these clusters.

\section{The strains}

The well-established species. Our collection of strains includes representatives of species established by conventional methods. It is useful to present the results of numerical studies with these strains before considering the results with other strains.

Streptococcus agalactiae (Lancefield group B) has a number of distinctive properties, including ability to grow on $40 \%$ bile agar, growth in $4 \%$ but not $6.5 \% \mathrm{NaCl}$ broth, ability to hydrolyse hippurate and give the CAMP reaction (Munch-Petersen \& Christie, 1947). It is heat sensitive and does not split aesculin (Munch-Petersen, 1954). With the single-linkage method all 7 strains included formed one cluster as strains $\mathbf{I} 68$ to I74. With Harrison's method these same 7 strains are clustered at the $99 \%$ significance level, with the addition of one strain of Lancefield's group $\mathrm{G}$ which was the only strain clustered in Harrison's method in a way that caused surprise. Harrison's method was used with a double weighting on positive matches, and this strain of group $G$ does have some features in common with the group B strains, such as ability to grow on $40 \%$ bile, production of acid from glycerol aerobically, production of hyaluronidase and $\beta$-glucuronidase and the splitting of arginine, but it does not grow in salt broth, split hippurate, or give the CAMP reaction. In the single-linkage method the overall similarity of this strain to the 7 agalactiae strains was in all cases less than $70 \%$.

Streptococcus salivarius is also a distinctive species and if we wish to classify strains on the basis of a single distinctive character, levan production characterizes 


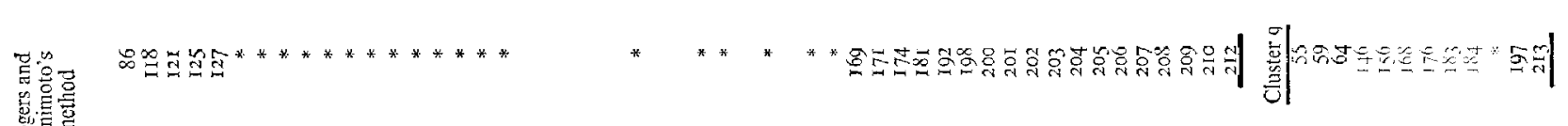

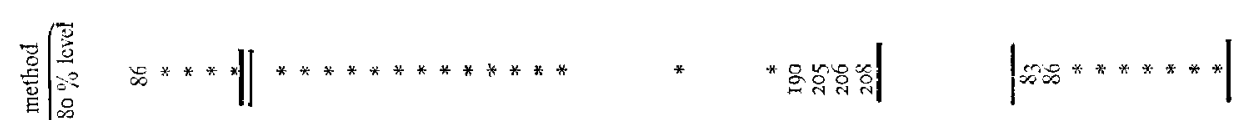

焉变

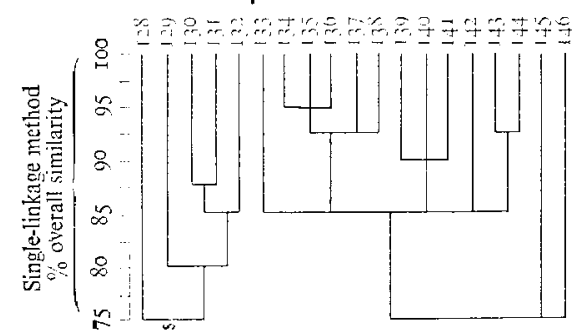

$|x * \cdots \cdots \cdots+|$

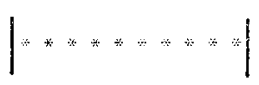

1

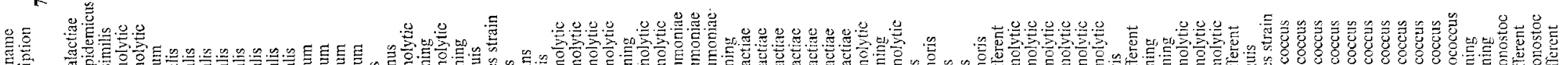

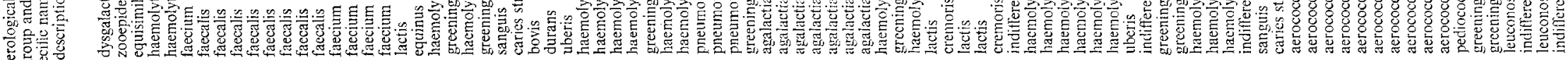

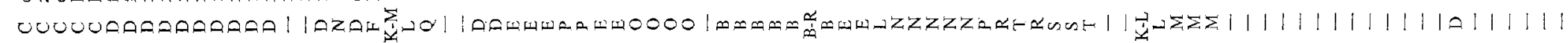

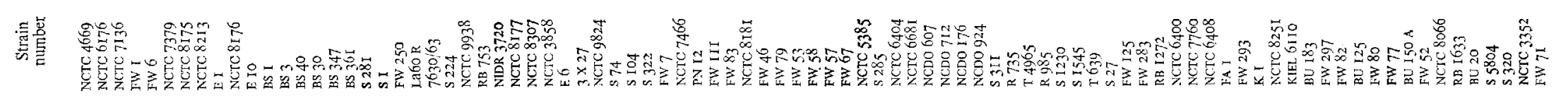

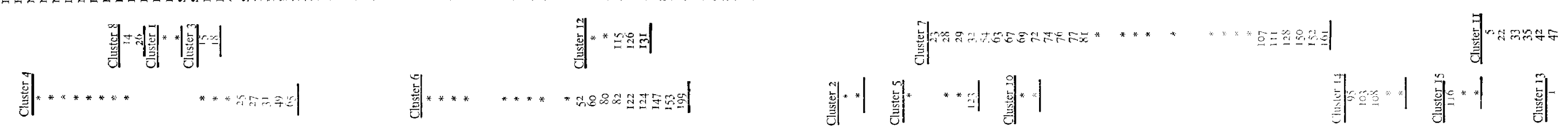

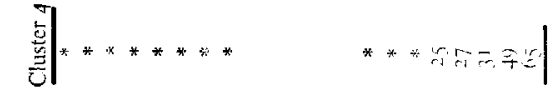

橧:

焉焉

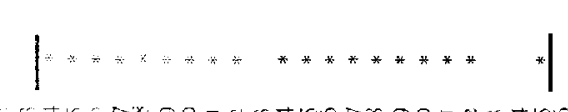

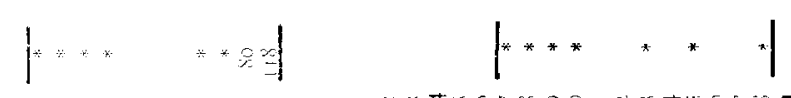

$\mid \cdots \ldots \ldots \ldots+\cdots$
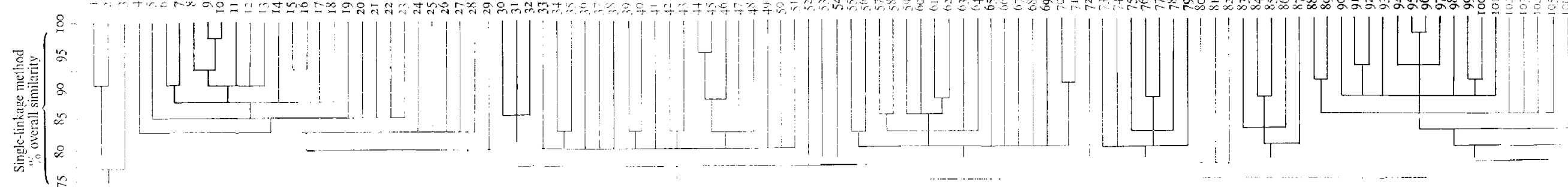

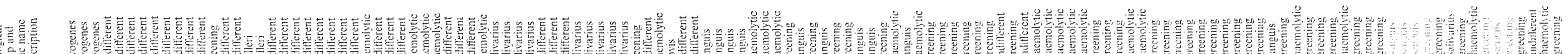

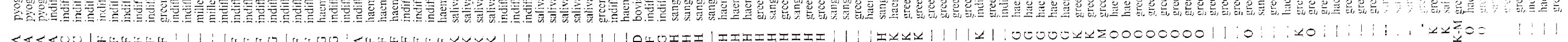

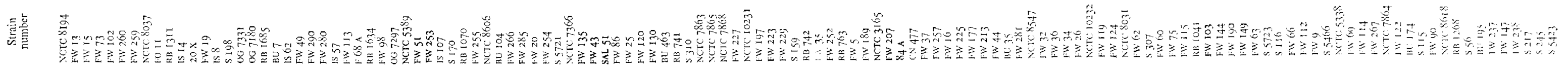


a reasonably homogeneous species (Williams, 1956). Ten salivarius strains are included and in the single-linkage method they are found as strains 37 to 40,44 to 48 , and strain I18. Strain II 8 has a similarity coefficient of greater than $70 \%$ with 6 of the other 9 strains. Nine of the Io salivarius strains are included in a cluster with other strains, and 5 of these form a discrete cluster at the $82.5 \%$ level. At the $99 \%$ probability level Harrison's method clusters 7 of these Io strains and includes in the cluster strain 80, a strain which does not produce levan but which is remarkably like the salivarius strains.

Streptococcus faecalis and other enteric streptococci have been studied previously by numerical methods. Colobert \& Blondeau (1962) examined a collection of enterococci which fulfilled Sherman's (1938) criteria. They examined I73 strains for the occurrence of 38 characters, and since their study was restricted to enterococci they included more tests of value for distinguishing these strains than was done in the present work. They found two major divisions, one containing varieties of $S$. faecalis, var. faecalis and var. liquefaciens, the other division almost forming a linear series with centres of population formed by $S$. faecium, $S$. durans, and some strains for which Colobert \& Blondeau propose the name $S$. innominatus. Raj \& Colwell (I966) examined 40 enterococci and 8 other streptococci and found all the enterococci to form a homogeneous cluster (they included $S$. bovis but excluded $S$. equinus from the enterococci). This finding could have been determined by their choice of tests which was heavily weighted by appearance in culture.

In the present work the single-linkage method formed, at the $85 \%$ level, a homogeneous cluster of Streptococcus faecalis and S. faecium strains, numbered I33 to I44. Harrison's method at the $99 \%$ probability level formed the same cluster with the addition of five strains, one strain each of $S$. faecium, serological group Q and $S$. durans, and two strains of aerococci which occurred in another cluster. There are more $S$. faecalis and $S$. faecium strains in this collection than $S$. durans, S. bovis and $S$. equinus strains and this is a possible reason why the faecalis and faecium strains were clustered together. However, they are very similar.

The aerococci are similar to the enterococci in a number of properties such as salt tolerance and bile tolerance. They can be mistaken for streptococci (Colman, 1967). The aerococci formed a discrete cluster by the single-linkage method, strains 200 to 207, and by Harrison's method, strains 200 to 209. As mentioned above, some strains by Harrison's method occurred in both the cluster of the aerococci and the enterococci.

There are within the present collection other strains that would generally be accepted as distinctive populations at a level equivalent to that of species. They appear only in the clusters formed with the single-linkage method because of the limit imposed on cluster size in Harrison's method. Three strains of Streptococcus pyogenes (Lancefield group A) are found clustered I to 3, five strains of Lancefield group $\mathrm{G}$ (haemolytic and large-colony variety) are clustered 83 to 87 , three haemolytic strains of Lancefield group $\mathrm{C}$ are clustered at 130 to 132 , and three strains of pneumococcus are clustered at I64 to $\mathrm{I} 66$.

Some proposed specific groupings. In describing the residues found in hydrolysates of whole cell walls it was noted by Colman \& Williams (1965) that some indifferent (non-haemolytic) streptococci of Lancefield groups A, C, and G differed from the more common haemolytic members of these serological groups and that their cell-wall residues were similar to those from indifferent streptococci of Lancefield's group F 
and from the strains called $S$. milleri (Guthof, 1956). All these strains show a number of similar physiological and biochemical reactions, such as resistance to bacitracin and nitrofurazone, ability to split arginine, production of acetoin from glucose, and ability to grow in $0.01 \%$ potassium tellurite. It is therefore not surprising that these strains should be classified together in both the single-linkage method and in Harrison's method. At the $85 \%$ similarity level in the single-linkage method the cluster was formed of the strains numbered 5 to 23 , and 17 of these strains occurred in the 19 strains clustered at the $99 \%$ probability level in Harrison's method. One of the strains added at the $82.5 \%$ level to the cluster in the single-linkage method was a representative of the 'minute' group G streptococci (Bliss, I937).

Another cluster, the strains numbered 55 to 69 , was made up of strains that either belonged to serological group $\mathbf{H}$ or produced a dextran-like polysaccharide from sucrose (the diagnostic character of $S$. sanguis; Hehre \& Neill, I946) or had both these characters. The strains in this cluster all contained readily detectable amounts of rhamnose in the cell wall and split arginine yielding ammonia, most produced acid from the sugars lactose, sucrose, trehalose and salicin, and most grew in the presence of $10 \%$ bile.

A third large cluster, strains 88 to I I 2, contained strains that either gave a precipitate with sera of the groups $\mathrm{O}, \mathrm{K}$, or $\mathrm{M}$, or else lack recognized 'Group' or 'Ottens' antigens. These strains had no readily detectable amounts of rhamnose in the cell wall and usually yielded the residue anhydroribitol in the cell-wall hydrolysates; most of the strains gave zones of greening on blood agar, few gave acid from trehalose and salicin, and none produced ammonia from arginine. These strains would usually be called Streptococcus viridans or S. mitis. The same set of characters was also possessed by strains I I 3 to I I5, which would usually be classified as $S$. sanguis; these three strains belong to the second serological type established among the $S$. sanguis strains by Washburn, White \& Niven (1946). If we classify solely on ability to produce a polysaccharide from sucrose these three strains would be called $S$. sanguis, but on overall similarity they belong with the strains numbered 88 to I I 2 .

Thus the results of the numerical studies suggest that the viridans-like streptococci of man contain three recognizable divisions in addition to the well-established species Streptococcus salivarius.

\section{DISCUSSION}

In so far as there is a formal classification of streptococci, it is based on a combination of physiological, biochemical and serological reactions. Some bacteriologists, in their enthusiasm for a diagnostic scheme that is excellent for the haemolytic streptococci originally included, have gone so far as to make serological grouping the sole basis of classification. I cannot accept this view and I shall later present evidence, some of which is apparent here, that a classification based solely on serological group will yield physiologically heterogeneous taxa in at least all the groups from A to $\mathrm{N}$. I believe that the definition of species should be based on the possession of a number of distinctive, independent and highly correlated attributes. Among the well-documented species isolated from man I suggest, on the basis of past experience, that Streptococcus agalactiae, $S$. pneumoniae, $S$. pyogenes, $S$. salivarius and $S$. faecalis and its varieties, fulfil these criteria.

There are many streptococci outside these well-defined species and it is among 
these that taxonomic confusion has reigned. While it is possible that they form a series with a common gene pool, it seemed worth applying the methods of numerical taxonomy to seek clusters of strains that possess characters highly correlated within the cluster, but which rarely occur together outside the cluster. Two of the three programmes used in this work produced clusters that conformed generally to the well-established taxa mentioned above, and they both produced approximately the same three divisions among the less well-known strains. The third clustering method, that devised by Rogers \& Tanimoto, produced clusters that seemed to be unrelated to the conventional classification of streptococci. This last method was originally used in a study of higher plants, for which its originators clearly found it to give satisfactory results; a possible reason for its failure in the present work may lie in the nature of the data; ours is largely binary in form; theirs was multi-state qualitative.

The three population nodes found among the viridans-like streptococci can usefully serve as the centres of aggregated species, which can be subdivided, if so desired for special purposes, into sub-species. The central node of one of these aggregated species contains representatives of the indifferent streptococci of Lancefield groups A, C, F and $\mathrm{G}$, some strains that contain antigens of the Ottens or Ottens \& Winkler series, and some strains that lack recognized antigens but which were called Streptococcus milleri by Guthof (1956). The central node of the second aggregated species contains representatives of the species $S$. sanguis and members of serological group $H$. The third central node contains representatives of the serological groups $\mathrm{O}, \mathrm{K}$, and $\mathrm{M}$, and this node can readily be distinguished from the other two because of the combination of unusual cell-wall composition and unusual biochemical reactions.

The data presented in this paper indicates strongly that if a bacterial population contains clusters of strains with attributes distributed in the non-random manner that is characteristic of a species, then numerical methods can demonstrate these clusters. However, evidence is also presented that these methods will only do this when the right programme is chosen.

This study was supported by the Medical Research Council, and generous help with the computer analyses was provided by International Business Machines, The Centre for Computing and Automation at Imperial College, Imperial Chemical Industries, The Rothamsted Experimental Station, Mr J. M. Faulks, Mr H. Gluck, Mr J. C. Gower and Mr P. J. Harrison.

\section{REFERENCES}

ANDERSON, A. B. \& HaRT, P. D. (1934). The lysis of pneumococci by sodium desoxycholate. Lancet ii, 359.

ANDREWES, F. W. (1930). Note on the fermentation of starch by certain haemolytic streptococci. J. Path. Bact. 33, I45.

AYERS, S. H. \& RUPP, P. (I922). Differentiation of hemolytic streptococci from human and bovine sources by hydrolysis of sodium hippurate. J. infect. Dis. 30, 388 .

Burss, E. A. (1937). Studies upon minute streptococci. III. Serological differentiation. J. Bact. 33, 625.

Colman, G. (1967). Aerococcus-like organisms isolated from human infections. J. clin. Path. 2o, 294.

Colman, G. \& Winliams, R. E. O. (1965). The cell walls of streptococci. J. gen. Microbiol. 4I, 375.

Colobert, L. \& BlondeaU, H. (1962). L'espèce Streptococcus faecalis. I. Étude de l'homogénéité par la méthode adansonnienne. Annls Inst. Pasteur, Paris I03, 345. 
DeIBEL, R. H. (1964). The group D streptococci. Bact. Rev. 28, 330.

GutHOF, O. (1956). Über pathogene 'vergrünende Streptokokken'. Streptokokken-Befunde bei dentogenen Abszessen und Infiltraten im Bereich der Mundhöhle. Zentbl. Bakt. (Abt. I. Orig.) I66, 553 .

Harrison, P. J. (1968). Cluster Analysis. Appl. Statist. (in the Press).

Hehre, E. J. \& NeILL, J. M. (I946). Formation of serologically reactive dextrans by streptococci from subacute bacterial endocarditis. J. exp. Med. 83, 147 .

Henriksen, S. D. \& JonER, P. E. (1962). Production of hyaluronic acid by a strain of Streptococcus zymogenes. Acta path. microbiol. scand. 56, 70.

MAYR, E. (1965). Numerical phenetics and taxonomic theory. Syst. Zool. 14, 73.

Munch-Petersen, E. (1954). Note on Streptococcus agalactiae. Int. Bull. bact. Nomencl. Taxon. 4, 129.

Munch-Petersen, E. \& Christie, R. (1947). On the effect of the interaction of staphylococcal $\beta$-toxin and group-B streptococcal substance on red blood corpuscles and its use as a test for the identification of Streptococcus agalactiae. J. Path. Bact. 59, 367.

RAJ, H. \& Colwell, R. R. (1966). Taxonomy of enterococci by computer analysis. Can.J. Microbiol. I2, 353 .

Rogers, D. J. \& TANimoto, T. (1960). A computer program for classifying plants. Science, N.Y. I32, III5.

Sherman, J. M. (1938). The enterococci and related streptococci. J. Bact. 35, 81.

Skadhauge, K. \& Perch, B. (1959). Studies on the relationship of some alpha-haemolytic streptococci of human origin to the Lancefield group M. Acta path. microbiol. scand. 46, 239.

SNEATH, P. H. A. (I962). The construction of taxonomic groups. Symp. Soc. gen. Microbiol. 12, 289.

Sokal, R. R. \& SNeath, P. H. A. (1963). Principles of Numerical Taxonomy, p. I8o. San Francisco and London: W. H. Freeman and Company.

Washburn, M. R., WhIte, J. C. \& Niven, C. F., Jr. (1946). Streptococcus s.b.e.: immunological characteristics. J. Bact. 5I, 723.

Williams, R.E.O. (1954). Glucuronidase production by serotypes of Streptococcus pyogenes. J. gen. Microbiol. 10, 337.

Williams, R. E. O. (1956). Streptococcus salivarius (vel hominis) and its relation to Lancefield's group K. J. Path. Bact. 72, 15.

Williams, R. E. O. (1958). Laboratory diagnosis of streptococcal infections. Bull. Wld Hith Org. I9, 153 . 